\title{
Comparison of intravenous digital subtraction angiography with radionuclide ventilation-perfusion lung scanning in patients with suspected pulmonary embolism
}

\author{
KC FLINT, N MCI JOHNSON, A MANNHIRE, P DAWSON, S GEORGE, PJ ELL \\ From the Medical Unit, the Department of Radiology, and the Institute of Nuclear Medicine, Middlesex \\ Hospital, London
}

ABSTRACT Fifty one patients with suspected pulmonary embolus were studied by radionuclide ventilation-perfusion scanning and intravenous digital subtraction pulmonary angiography. In 31 patients the results of both investigations were reported as negative and in a further 11 both results were reported as positive, giving a concordance rate of $82.3 \%$. In nine patients there was disagreement between the two investigations and an attempt to resolve this difference on the basis of clinical data was made. On this basis digital subtraction angiography would have falsely classified three patients as not having an embolus who were correctly identified by radioisotope scanning. Angiography would, however, have correctly classified four patients misclassified by ventilation-perfusion scanning. The relative merits of digital subtraction angiography and radionuclide lung scanning are discussed.

Confirmation of the diagnosis of pulmonary embolism remains a common but difficult clinical problem. ${ }^{12}$ Accurate and early diagnosis is important because of the considerable morbidity and mortality associated with both untreated embolisation ${ }^{34}$ and inappropriate anticoagulant treatment. ${ }^{56}$

Radionuclide pulmonary perfusion scans are sensitive but lack specificity. Many chronic cardiac and pulmonary disorders result in perfusion abnormalities indistinguishable from pulmonary embolism. ${ }^{78}$ With the addition of ventilation studies specificity can be improved, but in the absence of a characteristic pattern of mismatched defects diagnostic accuracy may be low..$^{90}$ Pulmonary angiography therefore continues to be the most specific test for the detection of pulmonary emboli. The associated morbidity $(1 \%)$ and mortality $(0.5 \%)$ have, however, precluded its use in most patients and it is usually reserved for those with life threatening complications in whom aggressive treatment is being considered. ${ }^{11} 12$

Address for correspondence: Dr N Johnson, Middlesex Hospital, London W1N 8AA. (Reprints will not be available.)

Accepted 22 February 1985
Digital subtraction angiography combines digital imaging with computer subtraction techniques to provide arterial images after intravenous injection of contrast medium. ${ }^{13}$ This technique will provide images of the pulmonary circulation after injection of contrast medium via a central venous catheter. ${ }^{14}$ It therefore avoids the additional risk of cardiac catheterisation, which is responsible for most of the complications and the deaths that follow conventional pulmonary angiography. In 31 patients who were subsequently investigated by pulmonary angiography ${ }^{15}$ digital subtraction angiography correctly identified 12 as having suffered pulmonary embolism and excluded embolic disease in a further 18 . The result was falsely positive on only one occasion. In another study ${ }^{16}$ the sensitivity of digital subtraction angiography after experimentally induced gelfoam embolisation in dogs was found to be $100 \%$ if defects in parenchymal staining during the capillary phase were taken into account. Even if the more specific criteria of demonstrating vessel cut off and intravascular filling defects are used, the method compares favourably in sensitivity $(75 \%)$ with radionuclide lung scanning.

We have compared intravenous digital subtrac- 
tion angiography with radionuclide ventilationperfusion lung scanning, which is the most frequently used investigative technique in patients with suspected pulmonary embolism.

\section{Methods}

Fifty one consecutive patients were studied. All were referred to the Institute of Nuclear Medicine at the Middlesex Hospital with the suspected diagnosis of pulmonary embolism and gave informed consent to additional investigation. Sixteen were hospital inpatients, 14 were outpatients and 21 were referred from outlying hospitals. The mean age of this population was 52 years, 26 were female, 21 were smokers and eight had spirometric evidence of airflow obstruction $\left(\mathrm{FEV}_{1} / \mathrm{FVC}\right.$ ratio $\left.<70 \%\right)$.

In all cases ventilation-perfusion lung scans and digital subtraction angiograms were performed within 24 hours of each other. Each was reported independently in the presence of a current chest radiograph but without knowledge of specific clinical details or the result of the alternative investigation. Digital subtraction angiograms were reported in groups of $15-20$ by two radiologists (AM and PD) in combination and ventilationperfusion lung scans were reported in similar groups by two nuclear medicine physicians (SG and PE). All patients were interviewed by an independent clinician $(\mathrm{KF})$ and clinical features recorded.

\section{VENTILATION-PERFUSION LUNG SCANS \\ Ventilation study}

A Nuclear Associates xenon lung function unit wàs used. Each patient inhaled $222 \mathrm{MBq}(6 \mathrm{mCi})$ xenon 133 and images were obtained of the wash in, equilibrium, and wash out phases. Three wash out images were obtained: 0-1 min, 1-3 min, 3-6 min.

Increasing image acquisition time during wash out increases the sensitivity for gas trapping. All images were obtained in a posteroanterior projection.

\section{Perfusion study}

We gave albumin macroaggregrates labelled with technetium $99 \mathrm{~m}$ (140 MBq (4 mCi)) intravenously to each patient. Six planar projections were acquired-posteroanterior, anteroposterior, right and left laterals, and right and left posterior obliques. All images were obtained with a gamma camera having a large field of view-either a General Electric Maxicamera 400 AT or a Nuclear Enterprises Mk V LF.

Ventilation-perfusion lung scans were reported according to conventional criteria. They were regarded as positive where there was one or more segmental or two or more subsegmental perfusion defects accompanied by a normal chest radiograph and normal ventilation, and where perfusion defects were substantially larger than any corresponding radiographic abnormality with normal ventilation.

\section{DIGITAL SUBTRACTION ANGIOGRAPHY}

Digital subtraction angiography was performed with a Technicare DR960 apparatus, which has a maximum field size of $22.5 \mathrm{~cm}$. The framing rate used was 1.25 per second with a matrix of $512 \times 512$ pixels.

An arm vein was selected in the antecubital fossa and a $5 \mathrm{Fr}$ catheter with side and end holes was introduced by the Seldinger technique after local anaesthesia. The catheter was advanced centrally until the tip was situated in the superior vena cava. On one occasion no suitable arm vein was available and the femoral vein was punctured and the catheter tip placed in the inferior vena cava. Forty millilitres of iohexol (Omnipaque 300) $300 \mathrm{mg}$ iodine/ml were injected at $25 \mathrm{ml}$ per second. The patient was instructed to hold his breath in inspiration and frames of the arterial, capillary, and venous phases were recorded for later examination.

A posteroanterior view of the right lung was obtained followed by a $25^{\circ}$ left anterior oblique view of the left lung, a $22.5 \mathrm{~cm}$ field being used. Further oblique views of suspicious areas were obtained after a further injection of contrast material with a $15 \mathrm{~cm}$ field for elucidating specific problems.

Our preliminary studies showed three characteristic abnormalities in digital subtraction angiograms: (1) Defects in the perfusion image during the capillary filling phase. During this phase of the digital subtraction angiogram, parenchymal staining is pronounced and areas of oligaemia are easily seen. Such defects may correspond to areas of radiographic abnormality (I) or they may not (II)-see figure 1 . (2) Abrupt termination of pulmonary arteries with diminished peripheral perfusion (III). (3) Filling defects visible within the pulmonary arteries (IV)-figure 2.

The last two abnormalities (III and IV), which are commonly seen in experimental embolisation of the pulmonary arteries, ${ }^{15}$ were taken to be specific for pulmonary embolism. The first type of abnormality (perfusion defects) may or may not represent pulmonary emboli. To improve the specificity of such defects they were interpreted in conjunction with a chest radiograph. Multiple perfusion defects not corresponding to areas of radiological abnormality (IIb) were reported as positive for pulmonary embolism. Defects corresponding to areas of radiographic abnormality (I) and single defects which did not correspond to such areas were reported as negative (IIa). 


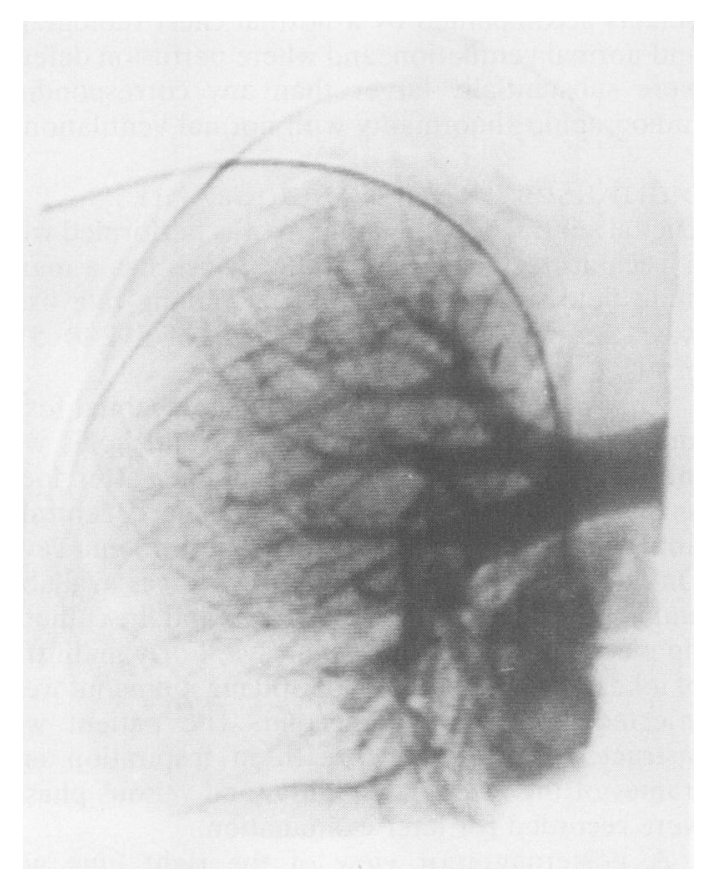

Fig 1 Digital subtraction angiogram, posteroanterior view, showing defect in parenchymal staining at right base.

Fig 2 Digital subtraction angiogram, right anterior oblique view, with $15 \mathrm{~cm}$ field showing filling defect in right basal branch of pulmonary artery (same patient as fig 1).

\section{Results}

The results of the 51 digital subtraction angiograms and ventilation-perfusion lung scans are compared in table 1 . In 31 patients the results of both investigations were reported negative and in 11 patients both results were reported as positive, giving a concordance rate of $82.3 \%$. In nine patients there was disagreement between the two results. The clinical features, digital subtraction angiographic findings, and results of radionuclide ventilation-perfusion lung scans of these patients are set out in table 2 . In two patients the final clinical diagnosis remained uncertain. If the final clinical diagnosis were to be accepted in those patients with strong clinical features, digital subtraction angiography would have falsely classified three patients as not having an embolism who were correctly identified as having one by ventilation-perfusion lung scanning. Angiography would, however, have correctly classified four patients misclassified by ventilation-perfusion lung scanning. In one patient with restrictive lung disease and abnormal ventilation and perfusion the angiogram was normal. Three patients were identified as having an embolism by digital subtraction angiography because of the specific finding of occlusion of a lower lobe artery. Two of these showed matched ventilation-perfusion abnormalities on radionuclide scans, presumably as the result of established pulmonary infarcts. In the third the clinical features suggested pulmonary embolism but the radionuclide perfusion scan was normal.

Of the 11 patients where both the angiograms and the ventilation-perfusion scans were positive, nine had a final clinical diagnosis that was in agreement. Where the results of both investigations were negative $(n=31)$, six had a final clinical diagnosis suggesting pulmonary embolism. All six were investigated 48 hours after the start of anticoagulant treatment.

Over the last year we have performed digital sub-

Table 1 Comparison of digital subtraction angiography (DSA) and radionuclide ventilation-perfusion $(V-Q)$ lung scanning in 51 patients with suspected pulmonary embolism

\begin{tabular}{llll}
\hline \multicolumn{2}{l}{ Digital subtraction angiography } & \multicolumn{2}{l}{ V-Q scan } \\
\cline { 3 - 4 } & & Negative & Positive \\
\hline \multirow{3}{*}{ Negative } & J Normal & 18 & 2 \\
& I* & 9 & 3 \\
Ila & 4 & 0 \\
Positive & Total & 31 & 5 \\
& IIb & 0 & 4 \\
III & 4 & 5 \\
IV & 0 & 2 \\
Total & 4 & 11 \\
\hline
\end{tabular}

"See under "Methods." 
Table 2 Clinical and radiographic features and findings of digital subtraction angiography (DSA) and of ventilation-perfusion lung scanning $(V-Q)$ in the nine patients in whom the two results disagreed

\begin{tabular}{|c|c|c|c|c|c|}
\hline $\begin{array}{l}\text { Age (y) } \\
\text { and sex }\end{array}$ & Clinical features & Radiography & $D S A$ & $V-Q$ & $\begin{array}{l}\text { Final } \\
\text { diagnosis }\end{array}$ \\
\hline $79 \mathrm{~F}$ & 1 y cough, dyspnoea & Kyphoscoliosis & NEGATIVE Normal & $\begin{array}{l}\text { POSITIVE Numerous } \\
\text { matched defects, single } \\
\text { unmatched defect }\end{array}$ & $\begin{array}{l}\text { Restrictive } \\
\text { lung } \\
\text { disease }\end{array}$ \\
\hline $50 \mathrm{~F}$ & $\begin{array}{l}48 \text { h pleuritic pain and } \\
\text { haemoptysis }\end{array}$ & Normal & NEGATIVE Normal & $\begin{array}{l}\text { POSITIVE Single left lower } \\
\text { zone segmental } \\
\text { perfusion defect }\end{array}$ & $\begin{array}{r}\text { Pulmonary } \\
\text { embolism }\end{array}$ \\
\hline $76 \mathrm{~F}$ & $\begin{array}{l}\text { Non-pleuritic chest pain, } \\
\text { dyspnoea }\end{array}$ & Normal & NEGATIVE Normal & $\begin{array}{l}\text { POSITIVE Single left apical } \\
\text { perfusion defect }\end{array}$ & Equivocal \\
\hline $21 \mathrm{~F}$ & $\begin{array}{l}\text { Pleuritic chest pain and } \\
\text { haemoptysis } 1 \text { w after } \\
\text { transatlantic flight }\end{array}$ & $\begin{array}{l}\text { Left lower zone } \\
\text { consolidation }\end{array}$ & $\begin{array}{l}\text { NEGATIVE Diminished } \\
\text { perfusion left lower } \\
\text { zone }\end{array}$ & $\begin{array}{l}\text { POSITIVE Multiple small } \\
\text { unmatched perfusion } \\
\text { defects }\end{array}$ & $\begin{array}{l}\text { Pulmonary } \\
\text { embolism }\end{array}$ \\
\hline $61 \mathrm{M}$ & $\begin{array}{l}\text { Pleuritic chest pain and } \\
\text { dyspnoea } 1 \mathrm{w} \text { after } \\
\text { right hip replacement }\end{array}$ & Normal & NEGATIVE Normal & $\begin{array}{l}\text { POSITIVE Multiple small } \\
\text { unmatched defects of } \\
\text { perfusion }\end{array}$ & $\begin{array}{l}\text { Pulmonary } \\
\text { embolism }\end{array}$ \\
\hline $69 \mathrm{M}$ & $\begin{array}{l}\text { Pleuritic chest pain, } \\
\text { dyspnoea, cough, } \\
\text { haemoptysis after } \\
\text { myocardial ischaemic } \\
\text { episode }\end{array}$ & $\begin{array}{l}\text { Right lower zone } \\
\text { consolidation }\end{array}$ & $\begin{array}{l}\text { POSITIVE Arterial cut off } \\
\text { and diminished } \\
\text { perfusion right lower } \\
\text { lobe }\end{array}$ & $\begin{array}{l}\text { NEGATIVE Matched right } \\
\text { lower zone defect }\end{array}$ & $\begin{array}{l}\text { Pulmonary } \\
\text { embolism }\end{array}$ \\
\hline $69 \mathrm{M}$ & $\begin{array}{l}\text { Left deep venous } \\
\text { thrombosis, dyspnoea, } \\
\text { pleuritic chest pain }\end{array}$ & $\begin{array}{l}\text { Right lower zone } \\
\text { consolidation }\end{array}$ & $\begin{array}{l}\text { POSITIVE Arterial cut off } \\
\text { and diminished } \\
\text { perfusion right lower } \\
\text { lobe }\end{array}$ & $\begin{array}{l}\text { NEGATIVE Matched right } \\
\text { lower zone defect }\end{array}$ & $\begin{array}{l}\text { Pulmonary } \\
\text { embolism }\end{array}$ \\
\hline $80 \mathrm{~F}$ & 4 m dyspnoea, cough & $\begin{array}{l}\text { Raised right } \\
\text { hemidiaphragm }\end{array}$ & $\begin{array}{l}\text { POSITIVE Arterial cut off } \\
\text { and diminished } \\
\text { perfusion right lower } \\
\text { zone }\end{array}$ & $\begin{array}{l}\text { NEGATIVE Right basal } \\
\text { perfusion defect with } \\
\text { gas trapping }\end{array}$ & Equivocal \\
\hline $30 \mathrm{~F}$ & $\begin{array}{l}\text { Iliac vein thrombosis, } \\
\text { sudden onset of } \\
\text { dyspnoea }\end{array}$ & Normal & $\begin{array}{l}\text { Positive Arterial cut off } \\
\text { and diminished } \\
\text { perfusion left lower } \\
\text { zone }\end{array}$ & NEGATIVE Normal & $\begin{array}{l}\text { Pulmonary } \\
\text { embolism }\end{array}$ \\
\hline
\end{tabular}

traction pulmonary angiography in three seriously ill patients from the intensive therapy unit. All were too ill for ventilation-perfusion scans or any attempt at a breath holding manoeuvre: nevertheless images of diagnostic quality were obtained. In two of these the presence and distribution of emboli was subsequently confirmed at postmortem examination.

\section{Discussion}

In our 51 subjects digital subtraction angiography proved a satisfactory method of imaging the pulmonary vasculature. The diagnostic information provided was in close agreement with that provided by the conventional diagnostic technique of ventilation-perfusion lung scanning (concordance $82.3 \%$ )

Our study was performed in an unselected group of patients in whom the physicians concerned with their care suspected pulmonary embolism. Many were outpatients and others had been referred from outlying hospitals, to return the same day. In view of the significant morbidity $(1.0 \%)$ and mortality $(0.5 \%)$ rates associated with pulmonary angiography, ${ }^{12}$ we did not think it ethically justifiable to proceed with this investigation in these patients. In the absence of the absolute standard of pulmonary angiography, we cannot be certain which of those patients in whom the results of the two investigations disagreed had suffered pulmonary embolism. In the absence of such an absolute standard an attempt was made to look at the two results in the light of the final clinical diagnosis. If this clinical diagnosis is accepted digital subtraction angiography would have falsely classified as not having embolism three patients identified as having it by ventilationperfusion scanning. All three of these patients had small peripheral perfusion defects on the radionuclide scan that were not seen on the angiogram. Digital subtraction angiography would, however, have correctly classified four patients misclassified by radionuclide lung scanning. One of these patients had pre-existing lung disease, in which the specificity of ventilation-perfusion lung scans is often low. ${ }^{9}$ In another two patients with the firm clinical diagnosis of pulmonary embolism digital subtraction angiography showed occlusion of the right lower lobe artery. The ventilation-perfusion scan demonstrated a matched right lower lobe defect, presumably the result of an established pulmonary infarct. Digital subtraction angiography will therefore identify those patients with established pulmonary infarcts and matched ventilation-perfusion abnormalities on radionuclide lung scans and there may be specific features on the angiogram confirming pulmonary embolism in those patients with pre-existing lung 
disease in whom ventilation-perfusion lung scans may not be diagnostic. The sensitivity of digital subtraction angiography at the present time, however, is such that it may miss very small peripheral perfusion defects that are visible on radionuclide perfusion scans.

Digital subtraction angiography with its requirement for central placement of the venous catheter is more invasive than radionuclide lung scanning. In our hands it has, however, proved an acceptable and well tolerated procedure. There were no complications in these patients, nor have there been any in nearly 700 patients having digital subtraction angiography for other reasons. The requirement for a 10 second breath holding period is the major limiting factor but with adequate explanation this can be achieved by all except those with the most severe cardiorespiratory disorders. Even without a breath holding manoeuvre adequate images may often be obtained in circumstances where ventilationperfusion scans would not be possible, such as in severely ill patients.

We have found it easier to arrange digital subtraction pulmonary angiograms at short notice, particularly overnight and at weekends. Radiology departments are normally well integrated with hospital facilities and radiologists are normally on call at all times. Nuclear medicine departments tend to be more remote and may be staffed only during office hours. The provision of radionuclide scans is often further limited by the availability of radioisotopes. The costs of the two techniques are difficult to compare directly as they will depend on the exact specifications of the equipment. The initial cost of the equipment for digital subtraction angiography in our hospital was about $£ 300000$, compared with about $£ 200000$ for ventilation-perfusion lung scanning. Consumables for a digital subtraction angiogram are slightly more expensive than for a radionuclide scan (about $£ 25$ versus $£ 8$ ) but the requirement in terms of staff time is thought to be roughly equivalent. Both digital subtraction angiography and radionuclide lung scanning equipment is used for a wide variety of other diagnostic investigations and the availability of one or other technique in any particular centre is likely to depend on the wider use of the equipment. In centres where both digital subtraction angiography and radionuclide scanning are available ventilation-perfusion lung scanning is likely to remain the investigation of first choice. In cases where radionuclide lung scans are not diagnostic digital subtraction angiography may sometimes provide a definitive diagnosis.

\section{References}

1 Sutton GC, Honey M, Gibson RV. Clinical diagnosis of acute massive pulmonary embolism. Lancet 1969;i:271-3.

2 Rosenow EC, Osmundsen PJ, Brown ML. Pulmonary embolism. Mayo Clin Proc 1981;56:161-78.

3 Barrit DW, Jordan SC. Anticoagulant drugs in the treatment of pulmonary embolism: controlled trial. Lancet 1960; i:1309-12.

4 Dalen JE, Alpert JS. Natural history of pulmonary embolism. Progr Cardiovasc Dis 1975;17:257.

5 Salzman EW, Deykin D, Shapiro RM, Rosenblatt R. Management of heparin therapy: controlled prospective trial. N Engl J Med 1975;292:1046-50.

6 Mersky C, Drapin A. Anticoagulant therapy. Blood 1965;25:567-9.

7 Secker-Walker RH, Siegal BA. The use of nuclear medicine in the diagnosis of lung disease. Radiol Clin North Am 1973;11:215-41.

8 Kelly MJ, Elliott LP. The radiological evaluation of patients with suspected pulmonary thrombo-embolic disease. Med Clin North Am 1974;59:3-6.

9 Robin ED. Over treatment of pulmonary embolism: the emperor may have no clothes. Ann Intern Med 1977;87:775-81.

10 McNeil BJ. A diagnostic strategy using ventilation/ perfusion studies in patients suspected for pulmonary embolism. J Nucl Med 1976;17:613-6.

11 Bell WR, Simon TL. A comparative analysis of pulmonary perfusion scans with pulmonary angiograms. $A m$ Heart J 1976;92:700-6.

12 Dalen JE, Brooks HL, Johnson LW, Meisher SG, Szucs MM, Dexher L. Pulmonary angiography in acute pulmonary embolism: indications, techniques and results in 367 patients. Am Heart J 1971;81:175-85.

13 Nudelman S, Capp MP, Fisher HP III, Frost MM, Roehrig H. Photoelectronic imaging for diagnostic radiology and the digital computer. Proceedings of the Society of Photo-optical Instrumentation Engineers $1979 ; 164: 136-46$.

14 Meaney TF, Weinstein MA, Buonocore E, et al. Digital subtraction angiography of the human cardiovascular system. Am J Roentgenol 1980;135:1153-60.

15 Reilly RF, Smith CW, Price RR, Patton JA, Diggs J. Digital subtraction angiography: limitations for the detection of pulmonary embolism. Radiology 1983; 149:379-82.

16 Pond GD, Ovitt TW, Capp MP. Comparison of conventional pulmonary angiography with intravenous digital subtraction angiography for pulmonary embolic disease. Radiology 1983;147:345-50. 